\title{
Nanoscale energy deposition in glass by double ultrashort Gauss-Bessel pulses
}

\author{
Jesus del Hoyo ${ }^{1,2}$, Remi Meyer ${ }^{1}$, Luca Furfaro ${ }^{1}$ and Francois Courvoisier ${ }^{1}$ \\ 1. FEMTO-ST Institute, Univ. Bourgogne Franche-Comté, CNRS, 15B Avenue des Montboucons, 25030, Besançon Cedex, France \\ 2. Applied Optics Complutense Group, Optics Department, Universidad Complutense de Madrid, Facultad de Ciencias Físicas, Plaza de las \\ Ciencias, 1, Madrid, 28040 Spain
}

The main advantage of ultrafast laser-based processing techniques is the capability of structuring the material in three dimensions, even inside the bulk of transparent materials. It allows for a very precise control and versatility of the manufacturing process. The formation of high aspect ratio voids in glass with diameters in the hundreds of nanometres range using only a single ultrafast laser pulse is particularly useful for many applications. Drilling of high aspect ratio nanochannels has been particularly successful when using zeroth-order Bessel beams [1, 2]. However, depending on the material and illumination geometry, limitations arise on the maximal and minimal nanochannel diameter (Fig. 1a). Here, we demonstrate that double pulses drastically improve the efficiency of the drilling (Fig. 1b) and absorption measurement allow for deciphering the mechanism.

a)

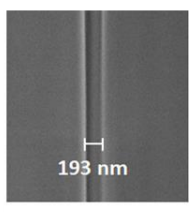

$3 \mu \mathrm{J}$

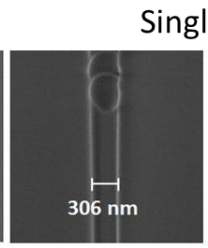

$5.2 \mu \mathrm{J}$
Single pulse

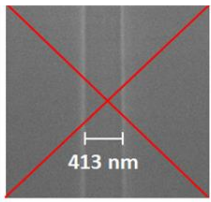

$6.3 \mu \mathrm{J}$

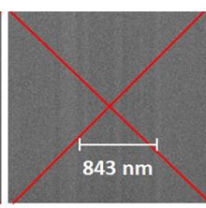

$8.5 \mu \mathrm{J}$

b)

Double pulse (delay $=500$ ps)

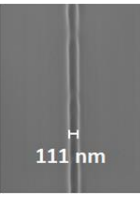

$2.0 \mu \mathrm{J}$

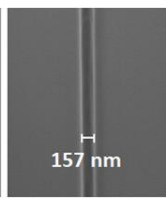

$3.4 \mu \mathrm{J}$

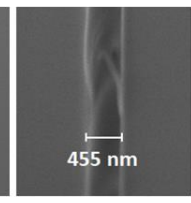

$8.8 \mu \mathrm{J}$

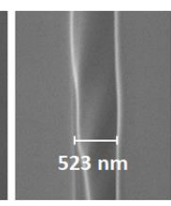

$12.9 \mu \mathrm{J}$

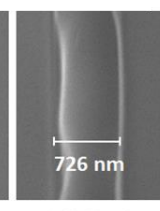

$16.5 \mu \mathrm{J}$ c)

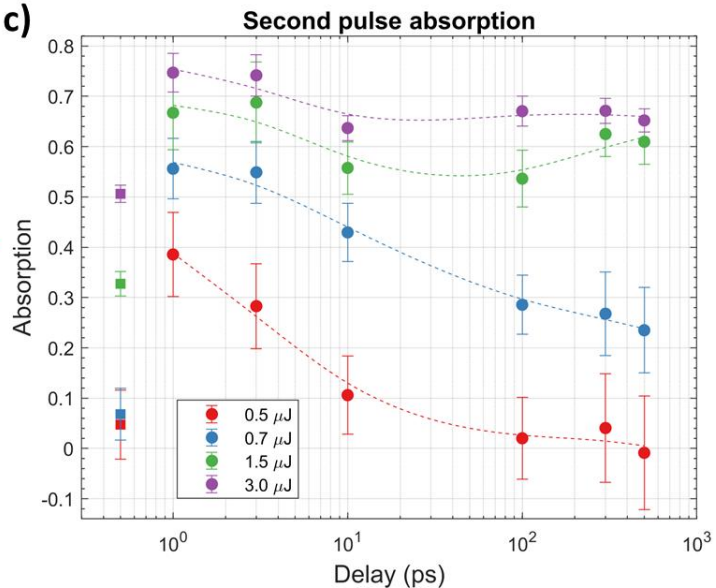

Fig. 1 Scanning electron microscopy (SEM) images of channels drilled using single pulses (a) and double pulses with a delay of $500 \mathrm{fs}$ (b).(c) Evolution of second pulse absorption as a function of time after the first pulse, for different input pulse energies. Square dots corresponds to the first pulse absorption.

Our experiment is based on splitting the input pulse energy into equal parts. We observed that for long inter-pulse delays (500 ps), the ability to form nanochannels is largely enhanced. Smaller structures than in single shot can be processed, down to $\sim 100 \mathrm{~nm}$ diameter. At high energies, wider voids channels are also drilled.

We have performed a detailed study of absorption [3]. Our results demonstrate for the first time that pulse splitting with a delay in the 100-500 ps allows for enhancing the confinement of energy deposition. Figure 1(c) shows the evolution in time of the absorption of the second pulse, for different input pulse energies. We observed that below a threshold energy, corresponding to channel opening, the absorption decreases in time (red and blue curves), as be expected from the formation of self-trapped excitons by the first pulse. In contrast, at higher energy, typically when channel opening occurs, the first pulse creates a high energy density state within $\sim 10$ ps, compatible with Warm Dense Matter. This state lasts at least 500 ps and highly absorbs the second pulse.

In conclusion, our results demonstrate that high intensity Bessel pulses create semi-metallic Warm Dense Matter, that highly efficiently absorbs a second pulse. The enhanced energy confinement increases the channel drilling efficiency. We believe that our results open new routes for laser processing of transparent materials, particularly for processing with burst in the $\mathrm{GHz}$ regime.

We acknowledge funding from the H2020 European Research Council (ERC), project 682032-PULSAR.

\section{References}

[1] M. K. Bhuyan, F. Courvoisier, P.-A. Lacourt, M. Jacquot, R. Salut, L. Furfaro, and J. M. Dudley, "High aspect ratio nanochannel machining using single shot femtosecond Bessel beams," App. Phys. Lett. 97, 081102 (2010).

[2] L. Rapp, R. Meyer, R. Giust, L. Furfaro, M. Jacquot, P.A. Lacourt, J. M. Dudley and F. Courvoisier, "High aspect ratio micro-explosions in the bulk of sapphire generated by femtosecond Bessel beams," Sci. Rep. 6, 34286 (2016).

[3] J. Hoyo, R. Meyer, L. Furfaro and F. Courvoisier, "Nanoscale confinement of energy deposition in glass by double ultrafast Bessel pulses," Nanophotonics 1, 0457 (2020). 